\title{
Normed Topological Pseudovector Groups
}

\author{
Piotr Niemiec
}

Received: 25 January 2010 / Accepted: 8 November 2010 / Published online: 20 November 2010

(C) The Author(s) 2010. This article is published with open access at Springerlink.com

\begin{abstract}
A normed topological pseudovector group (NTPVG for short) is a valued topological group $(V,+,\|\cdot\|)$ (not necessarily Abelian) endowed with a continuous scalar multiplication $\mathbb{R}_{+} \times V \ni(t, x) \mapsto t \cdot x \in V$ such that $0 \cdot x=e$ (e denotes the neutral element of $V), 1 \cdot x=x,(s t) \cdot x=s \cdot(t \cdot x), t \cdot(x+y)=(t \cdot x)+(t \cdot y)$ and $\|t \cdot x\|=t\|x\|$ for each $t, s \in \mathbb{R}_{+}$and $x, y \in V$. It is shown that every valued topological group can be isometrically and group-homomorphically embedded in a NTPVG as a closed subset by means of a functor. Locally compact NTPV groups are fully classified. It is shown that the (unbounded) Urysohn universal metric space can be endowed with a structure of a NTPV group of exponent 2.
\end{abstract}

Keywords Normed vector spaces • Topological groups • Locally compact groups • Valued groups • Dynamical systems • Urysohn universal metric space

Mathematics Subject Classifications (2010) 22A99 • 46B99 • 46A99

Normed vector spaces are metrizable topological groups of a very special kind. One may say that these are groups 'modelled' on the additive group of real numbers. The possibility of multiplying elements of a group by reals in a homomorphic way and the convex structure of a group facilitate investigations of such groups and has many deep topological and geometric consequences. For example, every normed vector space is an absolute extensor for metric spaces ([5]; see also [2, Theorem II.3.1]), locally compact normed vector spaces are finite dimensional and are uniquely determined (up to topological group isomorphism) by their dimension, non-locally compact completely metrizable normed spaces (that is, infinitedimensional Banach spaces [9]) as topological spaces are also uniquely determined

P. Niemiec $(\bowtie)$

Institute of Mathematics, Jagiellonian University,

ul. Łojasiewicza 6, 30-348 Kraków, Poland

e-mail: piotr.niemiec@uj.edu.pl 
(up to homeomorphism) by their topological weight $[15,16]$. The specificity of the algebraic structure of such groups leads to unrepeatable (in case of other groups) results such as the Hahn-Banach theorem and causes that the class of metrizable topological groups which are (topologically and algebraically) embeddable into normed spaces is very poor. Thus, it seems to be useful for studying topological groups to mark out topological groups which 'look like' normed spaces but form a huge enough class so that every metrizable topological group can be embedded in one of them. The aim of the paper is to give an example of such topological groups, namely normed topological pseudovector groups, briefly NTPVG, (see Abstract, Definition 1.1 or Remark 1.2). We shall show that there is a functor which links every valued topological group $(G, p)$ with a NTPVG $(L[G], L[p])$ in such a way that $G$ is naturally embedded (as a valued topological group) in $L[G]$ as a closed total subset (i.e. $L[G]$ is the smallest closed pseudovector subgroup of $L[G]$ containing $G$ ) and every Lipschitz homomorphism $f: G \rightarrow H$ of $(G, p)$ into a valued topological group $(H, q)$ extends (uniquely) to a continuous linear homomorphism $L[f]: L[G] \rightarrow$ $L[H]$ with the same Lipschitz constant. In addition, the functor $L$ built by us preserves monomorphicity and density of images. By means of this operator we define an $L$-regular subgroup of a valued topological group $(G, p)$ (a closed normal subgroup $H$ of $G$ is $L$-regular iff the canonical embedding $L[G] / L[H] \rightarrow L[G / H]$ is onto) and prove that every closed normal subgroup of $G$ is $L$-regular provided $(G, p)$ is complete. Several fundamental theorems of the classical functional analysis can easily be adapted for NTPV groups (with almost the same proof). However, one should be careful with this-even elementary facts on vector spaces can fail to hold for PV groups (for example, for a convex subset $K$ of a NTPV Abelian group $(G,+$ ) and $x \in G$ the set $K+x$ may not be convex). This raises new problems which are trivially solved in normed vector spaces and causes that the classification of locally compact NTPV groups is more complicated than in case of normed vector spaces. Although every locally compact NTPV group is isomorphic, as a topological group, to $\mathbb{R}^{n}$, there are uncountably many pairwise non-isomorphic structures of NTPV groups on $\mathbb{R}^{n}$ and for some of them the norm is given inexplicitly. Fortunately, the full classification of all locally compact topological pseudovector groups is possible and this is done in Section 2.

The last issue discussed here concerns the so-called Urysohn universal metric space $\mathbb{U}[17]$. It is well known for mathematicians investigating this space that there is no Banach space isometric to $\mathbb{U}$ (which easily follows e.g. from Holmes' theorem [7], see also [11]). In the opposite, we have shown in [12] that $\mathbb{U}$ can be endowed with a unique (up to isometric group-isomorphism) structure of a valued group of exponent 2. Here we improve this result by showing that the Boolean Urysohn group admits a structure of a NTPVG.

We believe that NTPV groups will be a useful tool in theory of metrizable topological groups. For well understanding of the paper it is enough to know basic facts on metrizable and valued groups, e.g. the material of Chapter 1 of [1].

\section{Foundations}

In this paper $\mathbb{R}_{+}$denotes the set of all nonnegative reals. For a function $f: X \rightarrow Y$ between topological spaces $X$ and $Y$, im $f$ and $\overline{\operatorname{im}} f$ stand for, respectively, the image 
of $f$ and its closure (in $Y$ ). Whenever we use the term 'group' with no adjectives, we think of a group with no topology (or with the discrete one). All topological groups are assumed to be Hausdorff and vector spaces are over the field of real numbers. If $(G,+)$ is a (not necessarily Abelian) group with the neutral element $e$, a function $p: G \rightarrow \mathbb{R}_{+}$is said to be a value iff for each $x, y \in G$ :

$$
\begin{array}{ll}
\text { (V1) } & p(x)=0 \Longleftrightarrow x=e, \\
\text { (V2) } & p(-x)=p(x), \\
\text { (V3) } & p(x+y) \leqslant p(x)+p(y) .
\end{array}
$$

Every value $p$ on $G$ induces left- and right-invariant metrics (respectively) $d_{L}$ and $d_{R}$ on $G$, namely $d_{L}(x, y)=p(-x+y)$ and $d_{R}(x, y)=p(x-y)$. If the space $\left(G, d_{L}\right)$ (or, equivalently, if the space $\left.\left(G, d_{R}\right)\right)$ is a topological group, we call the value $p$ topological and $G$ a valued topological group. This is equivalent to:

$$
\lim _{n \rightarrow \infty} p\left(x_{n}\right)=0 \Longrightarrow \lim _{n \rightarrow \infty} p\left(v+x_{n}-v\right)=0
$$

for each $v \in G$. Note that every value on an Abelian group is automatically topological. If $(G, p)$ and $(H, q)$ are two valued topological groups, so is $(G \oplus H, p \oplus q)$ where $(p \oplus q)(x \oplus y)=p(x)+q(y)$ for each $x \in G$ and $y \in H$.

In what follows, we assume that $p$ is a topological value on a group $(G,+)$. The metrics $d_{L}$ and $d_{R}$ induce the same topology, which we shall denote by $\tau_{p}$. Moreover, $d_{L}$ is complete iff so is $d_{R}$ and in that case we say that $(G, p)$ is a complete valued topological group. A sequence $\left(x_{n}\right)_{n=1}^{\infty}$ of elements of $G$ converges to $x \in G$ iff $\lim _{n \rightarrow \infty} p\left(x_{n}-x\right)=0$, iff $\lim _{n \rightarrow \infty} p\left(-x_{n}+x\right)=0$. A homomorphism $f: G \rightarrow$ $H$ between $(G, p)$ and a valued topological group $(H, q)$ is said to be Lipschitz [isometric] if there is a constant $\lambda \geqslant 0$ such that $q(f(x)) \leqslant \lambda p(x)$ [if $q(f(x))=p(x)$ ] for each $x \in G$. The least such a constant $\lambda$ is denoted by $\operatorname{Lip}(f)$. Additionally we put

$$
\operatorname{Lip}^{-1}(f)=\sup \left\{\frac{\operatorname{dist}_{p}(x, \operatorname{ker} f)}{q(f(x))}: x \in G \backslash \operatorname{ker} f\right\} \in[0,+\infty]
$$

provided $f$ is nonzero and $\operatorname{Lip}^{-1}(f)=0$ otherwise. In the above formula $\operatorname{dist}_{p}(x, \operatorname{ker} f)$ is the number $\inf \{p(x-y): \quad y \in \operatorname{ker} f\}=\inf \{p(-x+y): \quad y \in \operatorname{ker} f\}$ and it is the distance of a point $x$ from the set ker $f$ with respect to the metric $d_{L}$ or, equivalently, with respect to $d_{R}$. Lipschitz homomorphisms are continuous but not conversely.

By a theorem of Birkhoff [3] and Kakutani [8], every metrizable topological group admits a topological value $p$ for which $\tau_{p}$ coincides with the given topology of the group.

A special kind of valued topological groups are normed topological pseudovector groups.

Definition 1.1 A pseudovector group (in short: a $P V G$ or a $P V$ group) is a triple $(G,+, \cdot)$ such that

(PVG1) $(G,+)$ is a group (not necessarily Abelian) and '.' is an action (multiplication) $\mathbb{R}_{+} \times G \rightarrow G$ such that for each $t, s \in \mathbb{R}_{+}$and $x, y \in G$ :

$(\mathrm{PVG} 2) \quad(t s) \cdot x=t \cdot(s \cdot x)$,

(PVG3) $t \cdot(x+y)=(t \cdot x)+(t \cdot y)$,

(PVG4) $0 \cdot x=e$ and $1 \cdot x=x$ where $e$ is the neutral element of $G$. 
Elements of a PV group are called pseudovectors.

A topological pseudovector group (briefly, a TPVG or a TPV group) is a quadruple $(G,+, \cdot, \tau)$ where $(G,+, \cdot)$ is a PVG and $\tau$ is a topology on $G$ such that $G$ is a topological group with respect to $\tau$ and the action '.' is continuous.

A normed pseudovector group (in short: a NPVG or a NPV group) is a quadruple $(G,+, \cdot,\|\cdot\|)$ where $(G,+, \cdot)$ is a PVG and $\|\cdot\|: G \rightarrow \mathbb{R}_{+}$is a topological value on $G$ such that for any $t \in \mathbb{R}_{+}$and $x \in G$,

(N1) $\|t \cdot x\|=t\|x\|$.

In the above case the value $\|\cdot\|$ is called a norm.

A normed topological pseudovector group (in short: a NTPVG or a NTPV group) is a NPV group $(G,+, \cdot,\|\cdot\|)$ such that

$$
\left(G,+, \cdot, \tau_{\|\cdot\|}\right) \text { is a TPVG. }
$$

We shall abbreviate the above notation writing that $G$ or $(G,\|\cdot\|)$ is a NTPVG (similarly for TPV and PV groups). For simplicity, we shall always denote the neutral element of any group by 0 .

Remark 1.2 Usually for non-Abelian groups the multiplicative notation is more reasonable. In such a case one should write $(t, x) \mapsto x^{t}$ instead of $(t, x) \mapsto t \cdot x$ for the action of a PVG.

If $G$ is a $(\mathrm{N})(\mathrm{T}) \mathrm{PV}$ group as in Definition 1.1, it suffices to put $t \cdot x=-((-t) \cdot x)$ for $t<0$ and $x \in G$ in order to obtain an action $\mathbb{R} \times G \rightarrow G$ with the same properties as the original ones.

Note also that there is a difference between topological PV groups and PV topological groups (the latter are just topological groups with an additional multiplication by nonnegative reals satisfying the axioms (PVG2)-(PVG4)). However, each TPV group is a topological group, by its definition.

Notice that there is a one-to-one correspondence between PV groups and pairs $(G, \mathbb{T})$ where $G$ is a group and $\mathbb{T}=\left\{T_{t}\right\}_{t \in \mathbb{R}}$ is a one-parameter group of algebraic automorphisms of $G$ (that is, $T_{0}$ is the identity map on $G$ and $T_{t+s}=T_{t} \circ T_{s}$ for each $t, s \in \mathbb{R})$. Indeed, if '. ' is the action of a PV group, then $T_{t}(x)=\exp (t) \cdot x$ defines a one-parameter group of automorphisms; and conversely, given $\left\{T_{t}\right\}_{t \in \mathbb{R}}$, it suffices to put $0 \cdot x=0$ and $t \cdot x=T_{\log (t)}(x)$ for $t>0$ to obtain an action of a PV group. More interesting is the case of TPV groups: in that case the group $\mathbb{T}$ is continuous, i.e. $\lim _{t \rightarrow s} T_{t}(x)=T_{s}(x)$ for each $s \in \mathbb{R}$ and $x \in G$, and $\lim _{t \rightarrow-\infty} T_{t}(x)=0$ for all $x \in G$. This leads to a dynamical system $\Phi: \mathbb{R} \times G \rightarrow G$ given by $\Phi(t, x)=T_{-t}(x)$ in which 0 is asymptotically stable. We shall use this observation in Section 2 to classify locally compact TPV groups.

In the same way as it is done in vector spaces one defines a $P V$ subgroup and a convex subset of a PV group. If $K$ is a subset of a PV group $G$, the linear span and the convex hull of $K$ in $G$ are denoted by lin $K$ and conv $K$, respectively. One may show that $\operatorname{lin} K=\left\{\sum_{j=1}^{n} t_{j} \cdot a_{j}: t_{1}, \ldots, t_{n} \in \mathbb{R}_{+}, a_{1}, \ldots, a_{n} \in K \cup(-K), n \geqslant 1\right\}$ (provided $K$ is nonempty) and conv $K=\left\{\sum_{j=1}^{n} t_{j} \cdot a_{j}: t_{1}, \ldots, t_{n} \geqslant 0, \sum_{j=1}^{n} t_{j}=1, a_{1}, \ldots, a_{n} \in\right.$ $K, n \geqslant 1$ \}. If, in addition, $G$ is a TPV, the closures of lin $K$ and conv $K$ are denoted by $\overline{\text { lin }} K$ and $\overline{\text { conv }} K$ (respectively). The reader will easily check that $\{0\}$ is a convex subset of any PV group $G$ while $\{x\}=\{0\}+x$ may not be convex for some $x \in G$. 
Let us make a general convention that all undefined notions and notations which are clear for vector spaces have an analogous meaning as in case of vector spaces. Additionally, we define a monothetic TPV group as a TPV group $G$ for which $G=\varlimsup\{a\}$ for some $a$. If the latter equality holds, $a$ is said to be a generator of $G$. If we change order in adjectives, we obtain a stronger property: a TPV monothetic group is a TPV group which is monothetic as a topological group. Clearly, the only monothetic TPV groups among topological vector spaces (up to isomorphism) are the real line and the trivial space.

Example 1.3 It is very easy to check (and well known) that each norm on a vector space is topological. It turns out that a counterpart of this for norms on pseudovector groups is false. For example, let $\mathfrak{B}$ be the set of all finite subsets of $(0,+\infty)$. $\mathfrak{B}$ is a standard Boolean ring with the symmetric difference ' $\oplus$ ' as an addition and the intersection ' $\cap$ ' as a multiplication. What is more, $(\mathfrak{B}, \oplus, \cdot)$ is a PV group with an action '.' defined as follows: $0 \cdot A=\varnothing$ and $t \cdot A=\{t a: a \in A\}$ for $t>0$ and $A \in$ $\mathfrak{B}$. There are many 'classical' norms on $\mathfrak{B}$ which are not topological. For example, $\|A\|_{\infty}=\max A($ for $A \neq \varnothing)$ or $\|A\|_{p}=\left(\sum_{a \in A} a^{p}\right)^{1 / p}$ with $p \in[1,+\infty)$.

The following simple result facilitates checking that a NPV group is a NTPVG (we underline here that norms on PV groups have to be topological values).

Proposition 1.4 Let $(G,\|\cdot\|)$ be a $N P V$ group and

$$
\mathcal{C}(G)=\left\{x \in G: \lim _{t \rightarrow 1^{-}}\|x-t \cdot x\|=0\right\} .
$$

Then $\mathrm{C}(G)$ is a closed PV subgroup of $G$ and $\mathrm{C}(G)$ is a NTPV group.

Proof Clearly, $0 \in \mathrm{C}(G)$ and $t \cdot x \in \mathcal{C}(G)$ provided $x \in \mathcal{C}(G)$ and $t \geqslant 0$. What is more, if $x, y \in \mathrm{C}(G)$, then, by (V4) and (V2), $\|-x-t \cdot(-x)\|=\|-(t \cdot x)+x\|=\|-$ $x+(x-t \cdot x)+x \| \rightarrow 0\left(t \rightarrow 1^{-}\right)$and similarly $\|(x+y)-t \cdot(x+y)\| \leqslant \| x+(y-t$. $y)-x\|+\| x-t \cdot x \| \rightarrow 0\left(t \rightarrow 1^{-}\right)$, which shows that $\mathcal{C}(G)$ is a group. Now we shall check that $\mathrm{e}(G)$ is closed in $G$. Let $x_{n} \in \mathcal{C}(G)$ be such that $x_{n} \rightarrow x \in G$. Take $\varepsilon>0$. There is $m$ such that $\left\|x_{m}-x\right\| \leqslant \frac{\varepsilon}{3}$. Since $x_{m} \in \mathcal{C}(G)$, there is $\delta \in$ $(0,1)$ such that $\left\|x_{m}-\lambda \cdot x_{m}\right\| \leqslant \frac{\varepsilon}{3}$ provided $1-\delta<\lambda<1$. But then, for such a $\lambda$, one has $\|x-\lambda \cdot x\| \leqslant\left\|x-x_{m}\right\|+\left\|x_{m}-\lambda \cdot x_{m}\right\|+\left\|\lambda \cdot\left(x_{m}-x\right)\right\| \leqslant 2\left\|x_{m}-x\right\|+$ $\left\|x_{m}-\lambda \cdot x_{m}\right\| \leqslant \varepsilon$, which gives $x \in \mathcal{C}(G)$. The verification that the function $\mathbb{R}_{+} \times$ $\mathcal{C}(G) \ni(t, x) \mapsto t \cdot x \in \mathcal{C}(G)$ is continuous is left as an exercise.

The elements of the PV subgroup $\mathcal{C}(G)$ of a NPV group $G$ given by Eq. 1.1 are said to be continuous pseudovectors. The above result implies that a NPV group $G$ is a NTPVG iff $G$ coincides with the closed PV subgroup of $G$ generated by all its continuous pseudovectors. Another important consequence of Proposition 1.4 and the fact that the completion of an Abelian group is an Abelian group as well is stated in the following

Corollary 1.5 If $(G,\|\cdot\|)$ is an Abelian NTPV group, so is its completion. 
It is also easily seen by the result of Klee [9] that if $(G,\|\cdot\|)$ is an Abelian NTPV group which is completely metrizable as a topological space, then the norm $\|\cdot\|$ is complete.

Example 1.6 Let $m$ be the Lebesgue measure on $\mathbb{R}$ and let $\mathfrak{B}\left(\mathbb{R}_{+}\right)$be the set of the equivalence classes of all Borel subsets of $\mathbb{R}_{+}$of finite Lebesgue measure under the equivalence relation of almost equality (that is, two Borel subsets $A$ and $B$ of $\mathbb{R}_{+}$are almost equal if $m(A \backslash B)=m(B \backslash A)=0)$. For $A \in \mathfrak{B}\left(\mathbb{R}_{+}\right)$and $t>0$ put $0 \cdot A=$ $\varnothing(=\{0 \cdot a: a \in A\}), t \cdot A=\{t \cdot a: a \in A\}$ and $\|A\|=m(A)$. It is easily seen that $\left(\mathfrak{B}\left(\mathbb{R}_{+}\right), \oplus, \cdot,\|\cdot\|\right)$ where ' $\oplus$ ' denotes the symmetric difference is a NPV group. We claim that $\mathfrak{B}\left(\mathbb{R}_{+}\right)$is a NTPVG. First note that

$$
\left(t_{1} \cdot I\right) \oplus \ldots \oplus\left(t_{2 n} \cdot I\right)=\bigcup_{j=1}^{n}\left[t_{2 j-1}, t_{2 j}\right)
$$

for $0 \leqslant t_{1}<\ldots<t_{2 n}$ and $I=[0,1)$ which yields that $\mathfrak{B}\left(\mathbb{R}_{+}\right)=\varlimsup$ in $\{I\}$. So, thanks to Proposition 1.4, it suffices to show that $I \in \mathcal{C}\left(\mathfrak{B}\left(\mathbb{R}_{+}\right)\right)$. But for $t \in[0,1)$ we have $\|I \oplus t \cdot I\|=\|[t, 1)\|=1-t$, which finishes the proof. Thus $\mathfrak{B}\left(\mathbb{R}_{+}\right)$is a monothetic complete NTPVG which is infinite-dimensional as a topological space.

Now let $\mathfrak{B}$ be as in Example 1.3. For $0<t_{1}<\ldots<t_{2 n}$ put $\left\|\left\{t_{1}, \ldots, t_{2 n}\right\}\right\|_{0}=$ $\sum_{j=1}^{n}\left(t_{2 j}-t_{2 j-1}\right)$ and $\left\|\left\{t_{1}, \ldots, t_{2 n-1}\right\}\right\|_{0}=t_{1}+\sum_{j=1}^{n-1}\left(t_{2 j+1}-t_{2 j}\right)$. It can be simply verified that the function $\mathfrak{B} \ni\left\{t_{1}, \ldots, t_{p}\right\} \mapsto\left(t_{1} \cdot I\right) \oplus \ldots \oplus\left(t_{p} \cdot I\right) \in \mathfrak{B}\left(\mathbb{R}_{+}\right)$is a well defined monomorphism such that $\left\|\left\{t_{1}, \ldots, t_{p}\right\}\right\|_{0}=\left\|\left(t_{1} \cdot I\right) \oplus \ldots \oplus\left(t_{p} \cdot I\right)\right\|$ (thanks to Eq. 1.2). We infer from this that $\|\cdot\|_{0}$ is a norm on $\mathfrak{B}$ and the completion of $\left(\mathfrak{B},\|\cdot\|_{0}\right)$ coincides with $\left(\mathfrak{B}\left(\mathbb{R}_{+}\right),\|\cdot\|\right)$. This fact shall be applied in the last section.

The proofs of the following results (for topological vector spaces) may be repeated for TPV groups:

(TH1) A linear homomorphism between two NTPV groups is continuous iff it is Lipschitz.

(TH2) If $(G,\|\cdot\|)$ is a NTPV group and $H$ is a closed PV normal subgroup of $G$, then $\|[u]\|_{\pi}=\inf \{\|v\|: \quad v-u \in H\}$ is a norm on the quotient group $G / H$, $G / H$ is a NTPVG and the canonical projection $\pi: G \rightarrow G / H$ sends the open unit ball of $G$ onto the open unit ball of $G / H$. If, in addition, the norm $\|\cdot\|$ is complete, so is $\|\cdot\|_{\pi}$.

(TH3) If $U$ is an open, convex and symmetric neighbourhood of 0 in a TPV group $G$, its Minkowski functional $p_{U}: G \ni x \mapsto \inf \left\{t>0: \frac{1}{t} \cdot x \in U\right\} \in \mathbb{R}_{+}$is a continuous seminorm on $G$. If the TPV group $G$ has a bounded and convex neighbourhood of 0 , it is normable.

(TH4) (Closed Graph Theorem) A linear homomorphism between two complete NTPV groups is continuous iff its graph is closed in the product space.

(TH5) (Open Mapping Theorem) A continuous linear epimorhism between two complete NTPV groups is an open mapping.

(TH6) (Inverse Mapping Theorem) If a continuous linear homomorphism between two complete NTPV groups is bijective, then its inverse is continuous as well. 
Example 1.7 As it is done in functional analysis, for a continuous linear homomorphism $\varphi$ between two NTPV groups one may define the norm of $\varphi$ as $\operatorname{Lip}(\varphi)$ (see (TH1)). However, the PV group $\mathcal{B}(G)$ of all continuous linear endomorphisms of an Abelian complete PV group $G$ equipped with such a norm may not be TPV. For example, let $\left(\mathfrak{B}\left(\mathbb{R}_{+}\right),\|\cdot\|\right)$ be as in Example 1.6. One shows that if $0 \leqslant t_{1}<$ $\ldots<t_{n}$ and $\varphi: \mathfrak{B}\left(\mathbb{R}_{+}\right) \rightarrow \mathfrak{B}\left(\mathbb{R}_{+}\right)$is given by $\varphi(A)=\left(t_{1} A\right) \oplus \ldots \oplus\left(t_{n} A\right)$, then $\varphi$ is a continuous linear homomorphism and

$$
\|\varphi\|=\sum_{j=1}^{n} t_{j},
$$

which simply implies that $\mathcal{B}\left(\mathfrak{B}\left(\mathbb{R}_{+}\right)\right)$is not a TPV group. Indeed, just note that if $\varphi$ is the identity homomorphism on $\mathfrak{B}\left(\mathbb{R}_{+}\right)$, then, thanks to Eq. 1.3, $\|\varphi-t \cdot \varphi\|=1+t$ for $0<t<1$ and therefore the multiplication in discontinuous in $\mathcal{B}\left(\mathfrak{B}\left(\mathbb{R}_{+}\right)\right)$.

The following problem might be interesting:

Is every normed topological pseudovector group an absolute retract for metric spaces?

\section{Locally Compact TPV Groups}

This section is devoted to the full classification (up to topological linear isomorphism) of locally compact TPV and NTPV groups. We begin with four examples of such PV groups. In all of them the defined groups are equipped with the natural topology of $\mathbb{R}^{n}$ or $\mathbb{C}^{n}$ and thus they are locally compact. Since the multiplication by 0 is trivial in PV groups, we shall only define a multiplication by positive reals.

(I) Let $\lambda$ be a complex number such that $\operatorname{Re} \lambda>0$ and $\operatorname{Im} \lambda \geqslant 0$. If $\lambda$ is real (i.e. if $\operatorname{Im} \lambda=0)$, put $G(\lambda, 1)=(\mathbb{R},+)$; otherwise let $G(\lambda, 1)=(\mathbb{C},+)$. For a positive real number $t$ and $x \in G(\lambda, 1)$, let $t * x=t^{\lambda} x$ (recall that $t^{\lambda}=$ $\exp (\lambda \log t))$. It is easy to check that $G(\lambda, 1)$ is a TPV group.

(IA) Now assume in (I) that $\operatorname{Re} \lambda \geqslant 1$ and put $\|x\|=|x|^{\frac{1}{\operatorname{Re} \lambda}}$ for $x \in G(\lambda, 1)$. We leave this as an exercise that $\|\cdot\|$ is a norm on $G(\lambda, 1)$ which induces the topology of this group.

(II) Let $\lambda$ be as in (I) and $n \geqslant 2$. Put $G(\lambda, n)=G(\lambda, 1)^{n}$ and for $t>$ 0 and $x_{1}, \ldots, x_{n} \in G(\lambda, 1)$ let $t *\left(x_{1}, \ldots, x_{n}\right)=\left(y_{1}, \ldots, y_{n}\right)$ where $y_{k}=$ $t^{\lambda} \sum_{j=1}^{k} \frac{(\log t)^{k-j}}{(k-j) !} x_{j}$. A straightforward calculation shows that $G(\lambda, n)$ is a PV group. What is more, it is a TPVG (because $\operatorname{Re} \lambda>0$ ).

(IIA) Now assume in (II) that $\operatorname{Re} \lambda>1$. We claim that $G(\lambda, n)$ is normable. To see this, we shall make use of (TH3). Note that-since this group is locally compact-a subset of $G(\lambda, n)$ is bounded (in the sense of the PV structure) iff its closure is compact. So, thanks to (TH3), it is enough to check that the convex hull (in the PV structure) of the Euclidean unit ball $B$ is 'naturally' bounded. To do that, observe that

$$
\lim _{t \rightarrow 0^{+}} t^{\operatorname{Re} \lambda-1}(-\log t)^{p}=0
$$


for $p=0, \ldots, n$ and thus there is a constant $M>0$ such that $t^{\operatorname{Re} \lambda-1}(-\log t)^{p} \leqslant$ $M$ for $p=0, \ldots, n$ and $t \in(0,1]$. Now if $t_{1}, \ldots, t_{p}>0, \sum_{j=1}^{p} t_{j}=1,\left(x_{1}^{(1)}\right.$, $\left.\ldots, x_{n}^{(1)}\right), \ldots,\left(x_{1}^{(p)}, \ldots, x_{n}^{(p)}\right) \in B$ and $\left(y_{1}, \ldots, y_{n}\right)=\sum_{q=1}^{p} t_{q} *\left(x_{1}^{(q)}, \ldots, x_{n}^{(q)}\right)$, then

$$
\begin{aligned}
\left|y_{k}\right| & \leqslant \sum_{q=1}^{p} t_{q}^{\operatorname{Re} \lambda} \sum_{j=1}^{k} \frac{\left(-\log t_{q}\right)^{k-j}}{(k-j) !}\left|x_{j}^{(q)}\right| \\
& \leqslant \sum_{q=1}^{p} t_{q} \sum_{j=1}^{k} t_{q}^{\operatorname{Re} \lambda-1}\left(-\log t_{q}\right)^{k-j} \leqslant n M,
\end{aligned}
$$

which finishes the proof.

The proof of the next lemma is left as a simple exercise.

Lemma 2.1 Let $A, B$ and $P$ be three real $n \times n$-matrices and let $P$ be invertible. Then $P e^{A \log t} x=e^{B \log t} P x$ for each $x \in \mathbb{R}^{n}$ and $t>0$ iff $B=P A P^{-1}$.

Now we are ready to classify locally compact (N)TPV groups.

Theorem 2.2 Every nontrivial locally compact TPV group $G$ is isomorphic (as a TPV group) to $G\left(\lambda_{1}, n_{1}\right) \times \ldots \times G\left(\lambda_{p}, n_{p}\right)$ (for some $p \geqslant 1, \lambda_{1}, \ldots, \lambda_{p}$ and $\left.n_{1}, \ldots, n_{p}\right)$ where each $G\left(\lambda_{j}, n_{j}\right)$ is of type (I) or (II). The number $p$ and the pairs $\left(\lambda_{1}, n_{1}\right), \ldots,\left(\lambda_{p}, n_{p}\right)$ are uniquely (up to permutation) determined by $G$. The $P V$ group $G$ is normable iff each $G\left(\lambda_{j}, n_{j}\right)$ is of type (IA) or (IIA).

Proof By [6], every nontrivial locally compact contractible topological group is isomorphic (as a topological group) to $\mathbb{R}^{n}$ for some (unique) $n \geqslant 1$. Therefore we may assume that $G=\mathbb{R}^{n}$. For $t \in \mathbb{R}$ let $T_{t}: \mathbb{R}^{n} \ni x \mapsto e^{t} * x \in \mathbb{R}^{n}$ (where ' $*$ ' is the action of the PV group $G$ ). $T_{t}$, as a continuous group-homomorphism, is linear in the natural sense. Thus $\left\{T_{t}\right\}_{t \in \mathbb{R}}$ is a continuous one-parameter group of $n \times n$-matrices. Now e.g. by [13, Theorem 1.2], there is an $n \times n$-matrix $B$ such that $T_{t}=e^{t B}$. This means that

$$
t * x=e^{B \log t} x
$$

for each $t>0$ and $x \in \mathbb{R}^{n}$. By the Jordan matrix decomposition theorem, there are fields $\mathbb{K}_{1}, \ldots, \mathbb{K}_{p}$ each of which is either $\mathbb{R}$ or $\mathbb{C}$, scalars $\lambda_{j} \in \mathbb{K}_{j}$ with $\operatorname{Im} \lambda_{j}>0$ provided $\mathbb{K}_{j}=\mathbb{C}(j=1, \ldots, p)$, natural numbers $n_{1}, \ldots, n_{p} \geqslant 1$ and an invertible $\mathbb{R}$-linear (in the natural sense) operator $U: \mathbb{R}^{n} \rightarrow \mathbb{K}_{1}^{n_{1}} \oplus \ldots \oplus \mathbb{K}_{p}^{n_{p}}$ such that $B^{\prime}=$ $U \circ B \circ U^{-1}$ is an $\mathbb{R}$-linear operator such that

$$
B^{\prime}(x)=\left(\begin{array}{ccccc}
\lambda_{j} & 0 & 0 & \ldots & 0 \\
1 & \lambda_{j} & 0 & \ldots & 0 \\
\vdots & \vdots & \ddots & \vdots & \vdots \\
0 & \ldots & 1 & \lambda_{j} & 0 \\
0 & \ldots & 0 & 1 & \lambda_{j}
\end{array}\right) \cdot x
$$


for $x \in \mathbb{K}_{j}^{n_{j}}(j=1, \ldots, p)$. Thanks to Lemma 2.1, we may assume that $B^{\prime}=B$. Now since $\lim _{t \rightarrow 0^{+}} t * x=0$, we infer from Eq. 2.1 and the well known facts on dynamical systems that $\operatorname{Re} \lambda_{j}>0$ for each $j$. Now Eq. 2.2 combined with Eq. 2.1 yields that each of the summands $\mathbb{K}_{j}^{n_{j}}$ is a PV subgroup of $G$ isomorphic to $G\left(\lambda_{j}, n_{j}\right)$ and thus $G$ is isomorphic to $G\left(\lambda_{1}, n_{1}\right) \times \ldots \times G\left(\lambda_{p}, n_{p}\right)$. The uniqueness of such a decomposition follows from Lemma 2.1 (note that continuous homomorphisms of the additive group $\mathbb{R}^{n}$ are linear) and the uniqueness of the Jordan matrix decomposition.

It remains to prove that if $G(\lambda, n)$ is normable, then $\operatorname{Re} \lambda \geqslant 1$ and that $\operatorname{Re} \lambda \neq$ 1 provided $n>1$. Suppose that $\|\cdot\|$ is a norm on the PV group $G(\lambda, n)$ which induces its topology. For simplicity put $\mathbb{K}=G(\lambda, 1)$ and $q=\operatorname{Re} \lambda$. Observe that $t *(w e)=t^{\lambda} w e$ for any $t>0$ and $w \in \mathbb{K}$, and $e=(0, \ldots, 0,1) \in G(\lambda, n)$. Since $\|\cdot\|$ is continuous, there is $M>0$ such that $\|w e\| \geqslant M$ for all $w \in \mathbb{K}$ with $|w|=1$. Further, for each natural number $k \geqslant 1$ take $\gamma_{k} \in \mathbb{K}$ with $\left|\gamma_{k}\right|=1$ such that $k^{\lambda / q} \gamma_{k}=k$. Then (by the triangle inequality and (N1)) we obtain: $k\|e\| \geqslant\|k e\|=\left\|\left(k^{1 / q}\right) *\left(\gamma_{k} e\right)\right\|=$ $k^{1 / q}\left\|\gamma_{k} e\right\| \geqslant k^{1 / q} M$. This yields that the sequence $\left(k^{1 / q-1}\right)_{k=1}^{\infty}$ is upper bounded and therefore $q \geqslant 1$.

Now assume, in addition, that $n>1$. Put $y^{(k)}=\left(y_{1}^{(k)}, \ldots, y_{n}^{(k)}\right)=\frac{1}{k} *(k v)$ with $v=(1,0, \ldots, 0) \in G(\lambda, n)$ and $k \geqslant 1$. We infer from the fact that $\|\cdot\|$ induces the topology of $G(\lambda, n)$ that the set $\{x \in G(\lambda, n):\|x\| \leqslant r\}$ is compact for each $r>0$. What is more, it is convex in the sense of the PV structure. So, the sequence $\left(y_{2}^{(k)}\right)_{k=1}^{\infty}$ is bounded. But $\left|y_{2}^{(k)}\right|=k^{1-q} \log k$ and therefore $q>1$.

The uniqueness of $p$ in the above result implies that the TPV groups $G(\lambda, n)$ of type (I) or (II) are irreducible (or simple); that is, there are no two closed nontrivial PV subgroups $G_{1}$ and $G_{2}$ of $G(\lambda, n)$ such that $G(\lambda, n)=G_{1} \oplus G_{2}$.

\section{Enlarging a Valued Topological Group to a NTPV Group}

In this section we shall show that to every valued topological group $(G, p)$ there corresponds a NTPV group ( $L[G], L[p]$ ) which contains $G$ as a closed subgroup (and $L[p]$ extends $p$ ) such that any Lipschitz group-homomorphism $f: G \rightarrow H$ of $(G, p)$ into a valued topological group $(H, q)$ is uniquely extendable to a Lipschitz linear homomorphism $L[f]: L[G] \rightarrow L[H]$. (By (TH1), only Lipschitz group-homomorphisms may be extended to continuous linear ones. However, every continuous homomorphism may be made Lipschitz by a simple change of the value of its domain $G$ without changing the topology of $G$, but then $L[G]$ will change even as a set.) It can be easily seen that the presented below functor $L[\cdot]$ may be built in the category of pointed metric spaces and Lipschitz maps between them. It is however not of our interest.

From now to the end of the section, all values on groups are assumed to be topological. Let $m$ be the Lebesgue measure on $\mathbb{R}$. For a valued topological group $(G, p)$ let $L[G]$ be the set of (equivalence classes under the relation of $m$-almost everywhere equality of) all Borel functions $u: \mathbb{R}_{+} \rightarrow G$ whose image is separable and $L[p](u)=$ $\int_{0}^{\infty} p(u(t)) \mathrm{d} t$ is finite. (It follows e.g. from theory of separable Souslin spaces that a Borel image of a separable complete metric space is always separable and thus our assumption of separability of the image of $u$ is superfluous. However, this property of $u$ is of great importance for us and we want to make the presentation of a functor 
as elementary as it is possible.) Additionally, let $L^{0}[G]$ consists of all members $u$ of $L[G]$ for which there are numbers $n \geqslant 1$ and $0=t_{0}<t_{1}<\ldots<t_{n}<+\infty$ such that $u$ is constant on each of the intervals $\left[t_{j-1}, t_{j}\right), j=1, \ldots, n$, and $\left.u\right|_{\left[t_{n},+\infty\right)} \equiv 0$. It is clear that $L[G]$ is a group with the pointwise addition, i.e. $(u+v)(t)=u(t)+v(t)$, that $L^{0}[G]$ is a subgroup of $L[G]$ and that $L[p]$ is a value on $L[G]$. We claim that $L[p]$ is topological. To see this, fix $u \in L[G]$ and take a sequence $\left(v_{n}\right)_{n=1}^{\infty}$ of members of $L[G]$ such that $\lim _{n \rightarrow \infty} L[p]\left(v_{n}\right)=0$. We may assume that $L[p]\left(v_{n}\right) \leqslant 2^{-n}$. Put $g: \mathbb{R}_{+} \ni t \mapsto \sum_{n=1} p\left(v_{n}(t)\right) \in[0,+\infty]$. By the Lebesgue monotone convergence theorem, $\int_{0}^{\infty} g(t) \mathrm{d} t \leqslant 1$, which implies that $g(t)<+\infty$ for almost all $t \in \mathbb{R}_{+}$. This combined with the fact that $p$ is topological yields that $\lim _{n \rightarrow \infty} p\left(u(t)+v_{n}(t)-u(t)\right)=0$ for almost all $t \in \mathbb{R}_{+}$. But $p\left(u(t)+v_{n}(t)-u(t)\right) \leqslant 2 p(u(t))+g(t)$ and the function $2 p \circ u+g$ is Lebesgue integrable. Therefore $\lim _{n \rightarrow \infty} \int_{0}^{\infty} p\left(u(t)+v_{n}(t)-u(t)\right) \mathrm{d} t=0$ (by the Lebesgue dominated convergence theorem) and hence $L[p]$ is topological.

For positive $s>0$ let $\kappa_{s}: \mathbb{R}_{+} \ni t \mapsto t / s \in \mathbb{R}_{+}$. We define an action of the multiplicative semigroup $\mathbb{R}_{+}$on $L[G]$ by $0 * u=0$ and $s * u=u \circ \kappa_{s}$ for $s>0$. Basicly,

$$
L[p](s * u)=s L[p](u)
$$

for each $s \in \mathbb{R}_{+}$and $u \in L[G]$. Equation 3.1 says that $L[p]$ is a norm on $L[G]$. Later we shall show that $(L[G], L[p])$ is a NTPVG.

For each $x \in G$ let $\delta_{x}$ be a member of $L^{0}[G]$ defined as follows: $\left.\delta_{x}\right|_{[0,1)} \equiv x$ and $\left.\delta_{x}\right|_{[1,+\infty)} \equiv 0$. One checks that the map $\delta_{G}:(G, p) \ni x \mapsto \delta_{x} \in(L[G], L[p])$ is isometric (with respect to the left-invariant metrics and the right-invariant ones as well) and its image is closed. We shall not distinguish between $x$ and $\delta_{x}$. Under such an identification we see that $G$ is a closed subgroup of $L[G]$ such that

(L1) $\operatorname{lin} G=L^{0}[G]$

(and that $L[p]$ extends $p$ ). Similarly, we shall naturally identify $L\left[G_{0}\right]$ with a PV subgroup of $L[G]$ for any subgroup $G_{0}$ of $G$. This means that $u \in L[G]$ belongs to $L\left[G_{0}\right]$ iff there is a Borel subset $B$ of $\mathbb{R}_{+}$such that $u(B) \subset G_{0}$ and $m\left(\mathbb{R}_{+} \backslash B\right)=0$.

Further, for a Lipschitz homomorphism $f:(G, p) \rightarrow(H, q)$ define $L[f]$ : $L[G] \rightarrow L[H]$ by $L[f](u)=f \circ u$. It is easy to check that $L[f]$ is a well defined homomorphism which extends $f$ and that $\operatorname{Lip}(L[f])=\operatorname{Lip}(f)$.

The following properties of the operator $L[\cdot]$ are left as exercises:

(L2) $L$ is a functor, i.e. $L\left[\operatorname{id}_{G}\right]=\operatorname{id}_{L[G]}$ and $L[g \circ f]=L[g] \circ L[f]$ for any Lipschitz homomorphisms $f: G \rightarrow H$ and $g: H \rightarrow K$.

(L3) $L^{0}[G]$ is dense in $L[G]$.

(L4) $L\left[\bar{G}_{0}\right]=\overline{L\left[G_{0}\right]}$ for each subgroup $G_{0}$ of $G\left(\bar{G}_{0}\right.$ is the closure of $G_{0}$ in $\left(G, \tau_{p}\right)$ and $\overline{L\left[G_{0}\right]}$ is the closure of $L\left[G_{0}\right]$ taken in $\left.\left(L[G], \tau_{L[p]}\right)\right) ; G_{0}$ is a normal subgroup of $G$ iff $L\left[G_{0}\right]$ is a normal subgroup of $L[G]$.

(L5) $L[p]$ is complete iff so is $p$.

(To prove the 'if' part, it suffices to check that a sequence $\left(u_{n}\right)_{n=1}^{\infty}$ of members of $L[G]$ with $L[p]\left(u_{n}-u_{n+1}\right) \leqslant \frac{1}{2^{n}}$ for all $n \geqslant 1$ is convergent. But for such a sequence the function $f: \mathbb{R}_{+} \ni t \mapsto \sum_{n=1}^{\infty} p\left(u_{n}(t)-u_{n+1}(t)\right) \in[0,+\infty]$ is Lebesgue integrable and thus $f(t)<+\infty$ for almost all $t$. Let $A$ be the set of all $t \in \mathbb{R}_{+}$for which $f(t)$ is finite. For each $t \in A$ the sequence $\left(u_{n}(t)\right)_{n=1}^{\infty}$ is fundamental and hence converges to some $u(t) \in G$. Put $u(t)=0$ for $t \in$ $\mathbb{R}_{+} \backslash A$. Then $u \in L[G]$ and $\left(u_{n}\right)_{n=1}^{\infty}$ converges to $u$.) 
(L6) $L[G]$ is Abelian iff so is $G$.

(L7) $L[p](u+v)=L[p](v+u)$ for each $u, v \in L[G]$ iff $p(x+y)=p(y+x)$ for any $x, y \in G$.

(L8) $L[f]$ is injective iff $f$ is injective. If $q(f(x))=a p(x)$ for each $x \in G$ and some $a>0$, then $\frac{1}{a} * L[f]$ is isometric.

(L9) $L[f]\left(L^{0}[G]\right)=L^{0}[\operatorname{im} f] \subset \operatorname{im} L[f] \subset L[\operatorname{im} f]$ and $H \cap \operatorname{im} L[f]=\operatorname{im} f$ for a Lipschitz homomorphism $f: G \rightarrow H$. In particular, the image of $L[f]$ is dense in $L[H]$ iff the image of $f$ is dense in $H$.

(L10) $\operatorname{ker} L[f]=L[\operatorname{ker} f]$ and $\operatorname{Lip}^{-1}(L[f])=\operatorname{Lip}^{-1}(f)$.

(L11) $(L[G \oplus H], L[p \oplus q])$ is naturally isometrically PV-isomorphic to $(L[G] \oplus$ $L[H], L[p] \oplus L[q])$.

Now we are able to give a simple proof that $(L[G], L[p])$ is a NTPVG for each valued topological group $(G, p)$. By (L1), (L3) and Proposition 1.4, it suffices to show that every element of $G$ is a continuous pseudovector. The verification of this is elementary and is left to the reader.

Notice that the point (L7) is related to the bi-uniformity of $G$ and $L[G]$ and the biinvariance of the corresponding left-invariant (or right-invariant) metrics on $G$ and $L[G]$. The point (L6) may be strengthened as follows:

Proposition 3.1 Let $(G, p)$ be a valued topological group. For a subset $X$ of $L[G]$ the following conditions are equivalent:

(i) $x+t * y=t * y+x$ for each $x, y \in X$ and $t>0$,

(ii) $\operatorname{lin} X$ is Abelian,

(iii) there is a closed Abelian subgroup $H$ of $G$ such that $X \subset L[H]$.

In the proof of Proposition 3.1 we shall apply Corollary 3.3 (whose proof is left as an exercise) which simply follows from the following theorem of real analysis known as the Lebesgue density theorem (see e.g. [4, Theorem 7.33]):

Lemma 3.2 If $A$ is a measurable subset of the real line, then the set $\tilde{A}$ consisting of all $t \in A$ such that the limit $\lim _{\delta \rightarrow 0^{+}} \frac{m(A \cap(t-\delta, t+\delta))}{2 \delta}$ exists and is equal to 1 and of all $t \in \mathbb{R} \backslash A$ for which the latter limit exists and is equal to 0 is Lebesgue measurable and $m(\mathbb{R} \backslash \tilde{A})=0$.

Corollary 3.3 If $A_{0}, \ldots, A_{n}(n \geqslant 1)$ are measurable subsets of $\mathbb{R}_{+}$of positive measure $m$, there are positive numbers $t_{1}, \ldots, t_{n}$ such that $m\left(A_{0} \cap\left(t_{1} A_{1}\right) \cap \ldots \cap\left(t_{n} A_{n}\right)\right)>0$.

Proof of Proposition 3.1 We only need to show that (iii) is implied by (i). Suppose that (i) holds and for any $u \in X$ let $R(u)$ be the so-called essential image of $u$. That is, $y \in R(u)$ iff $m\left(u^{-1}(V)\right)>0$ for each open neighbourhood $V$ of $y$ in $G(R(u)$ coincides with the support of the pushforward measure of $m$ under $u)$. Note that $R(u)$ is a closed subset of $G$ such that $m\left(\mathbb{R}_{+} \backslash u^{-1}(R(u))\right)=0$ (by the separability of the image of $u$ ). It is enough to check that for every two elements $u_{1}$ and $u_{2}$ of $X$ the set $R\left(u_{1}\right) \cup$ $R\left(u_{2}\right)$ consists of pairwise commuting elements of $G$. (Indeed, having this we may define $H$ as the closed subgroup of $G$ generated by the set $\bigcup_{u \in X} R(u)$.) We argue by contradiction. Suppose there are $a$ and $b$ in $R\left(u_{1}\right) \cup R\left(u_{2}\right)$ (for some $u_{1}, u_{2} \in X$ ) with $a+b \neq b+a$. This yields that there exist open neighbourhoods $U_{a}$ and $U_{b}$ of $a$ and 
$b$, respectively, such that $x+y \neq y+x$ for any $x \in U_{a}$ and $y \in U_{b}$. Let $j, k \in\{1,2\}$ be such that $a \in R\left(u_{j}\right)$ and $b \in R\left(u_{k}\right)$. Put $A=u_{j}^{-1}\left(U_{a}\right)$ and $B=u_{k}^{-1}\left(U_{b}\right)$. Observe that the sets $A$ and $B$ are measurable and of positive measure $m$. So, by Corollary 3.3, there is $s>0$ such that $m(A \cap(s B))>0$. Now if $t \in A \cap(s B)$, then $u_{j}(t) \in U_{a}$ and $\left(s * u_{k}\right)(t) \in U_{b}$. Hence $u_{j}+s * u_{k} \neq s * u_{k}+u_{j}$, contradictory to (i).

Our next aim is to characterize all Lipschitz homomorphisms $f: G \rightarrow H$ for which $\operatorname{im} L[f]=L[\operatorname{im} f]$. We shall do this with aid of the so-called L-regular subgroups. In order to define them, we need to make some notes.

Assume that $H$ is a closed normal subgroup of a valued topological group $(G, p)$. Let $p_{\pi}$ be the (topological) value on $G / H$ induced by $p$ and $\pi:(G, p) \rightarrow\left(G / H, p_{\pi}\right)$ the canonical mapping (that is, $\left.p_{\pi}(\pi(x))=\inf \{p(y): y \in G, \pi(y)=\pi(x)\}\right)$. Since $\pi$ is Lipschitz, $L[\pi]: L[G] \rightarrow L[G / H]$ is well defined and $\operatorname{ker} L[\pi]=L[H]$ (by (L10)). The latter relation (together with (L4)) implies that there is a unique homomorphism $j_{H}: L[G] / L[H] \rightarrow L[G / H]$ such that $j_{H}(u+L[H])=L[\pi](u)$ for any $u \in L[G]$. It is easy to check that $j_{H}$ is linear. What is more, it is isometric, that is,

$$
L\left[p_{\pi}\right]\left(j_{H}(u+L[H])\right)=\|u+L[H]\|
$$

where $\|u+L[H]\|=\inf \{L[p](v): v \in L[G], v-u \in L[H]\}$ (compare with (TH2)). (To prove Eq. 3.2, simply check that $\operatorname{Lip}\left(j_{H}\right) \leqslant 1$ and that Eq. 3.2 holds for $u \in$ $L^{0}(G)$.) We call $j_{H}$ the canonical embedding. Its image is always dense, $j_{H}$ however may not be onto. This leads us to the following

Definition 3.4 A closed normal subgroup $H$ of a valued topological group $G$ is $L$ regular iff the canonical embedding $j_{H}: L[G] / L[H] \rightarrow L[G / H]$ is onto.

Observe that $H$ is $L$-regular iff $j_{H}$ has closed image, iff $L[\pi]$ is an epimorphism.

Since $j_{H}$ is isometric and isometries preserve completeness, (TH2) and (L5) yield

(L12) Every closed normal subgroup of a complete valued topological group is $L$ regular.

Utility of $L$-regularity is explained in the following

Proposition 3.5 For a Lipschitz homomorphism $f: G \rightarrow K$ between two valued topological groups $(G, p)$ and $(K, q)$ the following conditions are equivalent:

(i) $\operatorname{im} L[f]=L[\operatorname{im} f]$,

(ii) $\operatorname{Lip}^{-1}(f)<+\infty$ and ker $f$ is L-regular.

In particular, $L[f]$ is an epimorphism iff so is $f$ and (ii) holds.

Proof Put $H=\operatorname{ker} f$ and let $\pi: G \rightarrow G / H$ and $p_{\pi}$ be as in the note preceding Definition 3.4. There is a unique group-isomorphism $\tilde{f}: G / H \rightarrow \operatorname{im} f$ such that

$$
f=\tilde{f} \circ \pi .
$$

It can be easily verified that $\operatorname{Lip}(\tilde{f})=\operatorname{Lip}(f)$ and $\operatorname{Lip}\left(\tilde{f}^{-1}\right)=\operatorname{Lip}^{-1}(f)$. Thus, if (ii) holds, $\tilde{f}$ is a bi-Lipschitz isomorphism from $G / H$ onto im $f$ and therefore $L[\tilde{f}]$ is 
a linear isomorphism of $L[G / H]$ onto $L[\operatorname{im} f]$. But $L[f]=L[\tilde{f}] \circ L[\pi]$ and $L[\pi]$ is an epimorphism because of the $L$-regularity of $H$. This shows that (i) is implied by (ii).

To prove the converse implication, first we shall check that

$$
\operatorname{Lip}^{-1}(f)<+\infty
$$

provided $\operatorname{im} L[f]=L[\operatorname{im} f]$. Suppose, for the contrary, that Eq. 3.4 is false. This means that there is a sequence $\left(x_{n}\right)_{n=1}^{\infty}$ of elements of $G \backslash H$ such that $\operatorname{dist}_{p}\left(x_{n}, H\right) \geqslant$ $2^{n} q\left(f\left(x_{n}\right)\right)$. Put $t_{0}=0, t_{n}=t_{n-1}+\frac{1}{2^{n} q\left(f\left(x_{n}\right)\right)}$ for $n>0$ and $t_{\infty}=\lim _{n \rightarrow \infty} t_{n} \in[0,+\infty]$. Define $u: \mathbb{R}_{+} \rightarrow \operatorname{im} f$ by $\left.u\right|_{\left[t_{j-1}, t_{j}\right)} \equiv f\left(x_{j}\right)$ for each $j \geqslant 1$, and $\left.u\right|_{\left[t_{\infty},+\infty\right)} \equiv 0$ provided $t_{\infty}<+\infty$. It is easily checked that $u \in L[\mathrm{im} f]$ and therefore there is $v \in L[G]$ with $L[f](v)=u$. We infer from this that $f(v(t))=f\left(x_{j}\right)$ for almost all $t \in\left[t_{j-1}, t_{j}\right)$ and each $j \geqslant 1$. So, for these $t$ we obtain $v(t)-x_{j} \in \operatorname{ker} f=H$ and thus $p(v(t)) \geqslant$ $\operatorname{dist}_{p}\left(x_{j}, H\right) \geqslant 2^{n} q\left(f\left(x_{n}\right)\right)$. But then

$$
\begin{aligned}
\int_{0}^{\infty} p(v(t)) \mathrm{d} t & \geqslant \sum_{n=1}^{\infty} \int_{t_{n-1}}^{t_{n}} p(v(t)) \mathrm{d} t \geqslant \sum_{n=1}^{\infty} 2^{n} q\left(f\left(x_{n}\right)\right)\left(t_{n}-t_{n-1}\right)= \\
& =\sum_{n=1}^{\infty} 1=+\infty
\end{aligned}
$$

which denies the fact that $v \in L[G]$.

Now if Eq. 3.4 is fulfilled, the isomorphism $\tilde{f}$, appearing in Eq. 3.3, is bi-Lipschitz and therefore $L[\tilde{f}]$ is a linear isomorphism. We conclude from this, the relation $L[f]=L[\tilde{f}] \circ L[\pi]$ and the epimorphicity of $L[f]$ that $L[\pi]$ is an epimorphism as well, which finishes the proof.

Now we shall give a few illustrative examples dealing with the functor $L[\cdot]$.

\section{Example 3.6}

(A) Let $\mathbb{Z}_{2}=\{0,1\}=\mathbb{Z} / 2 \mathbb{Z}$ be the unique (up to isomorphism) group of cardinality 2 and let $p: \mathbb{Z}_{2} \rightarrow \mathbb{R}_{+}$be a value on $\mathbb{Z}_{2}$ given by $p(0)=0$ and $p(1)=1$. It is easily seen that $\left(L\left[\mathbb{Z}_{2}\right], L[p]\right)$ is naturally isometrically PV group-isomorphic to $\left(\mathfrak{B}\left(\mathbb{R}_{+}\right),\|\cdot\|\right)$ where the latter PV group is described in Example 1.6.

(B) To avoid misunderstandings, let us underline that in this example the term 'linear' (used with functions) is reserved for linear functions between vector spaces over the real field. Homomorphisms between PV groups will be called PVG-homomorphisms.

It is clear that $L[\mathbb{R}]$ is the classical Banach space $L^{1}(0,+\infty)$ of all real-valued Lebesgue integrable functions on $(0,+\infty)$. However, the PV structure of $L[\mathbb{R}]$ differs from the linear structure of $L^{1}(0,+\infty)$. With use of harmonic analysis, we are able to characterize all continuous PVG-endomorphisms of $L[\mathbb{R}]$. For this, let $\Phi: L[\mathbb{R}] \rightarrow L[\mathbb{R}]$ be a continuous group-homomorphism such that

$$
\Phi(s * u)=s * \Phi(u)
$$


for each $s>0$ and $u \in L[\mathbb{R}]$. Since the group $L[\mathbb{R}]$ is a vector space, $\Phi$ is linear. Let $G$ be the topological multiplicative group of positive real numbers. It can be simply verified that the measure $\lambda$ given by the formula $\mathrm{d} \lambda(t)=\frac{1}{t} \mathrm{~d} t$ is the Haar measure of $G$. Let $j$ denote the identity map on $G$. A straightforward calculation shows that the function $\Psi: L[\mathbb{R}] \ni u \mapsto u j \in L^{1}(\lambda)$ is a well defined (bijective) linear isometry. Now put $\Lambda=\Psi \circ \Phi \circ \Psi^{-1}: L^{1}(\lambda) \rightarrow L^{1}(\lambda) . \Lambda$ is a continuous linear operator such that $\Lambda\left(f_{s}\right)=(\Lambda f)_{s}$ for $s \in G$ and $f \in L^{1}(\lambda)$ where $g_{s}(t)=g(t / s)$ (this follows from Eq. 3.5). Now by [14, 3.8.4], there is a unique signed (real-valued) Borel measure $\mu$ on $G$ such that $\Lambda(f)=f * \mu$ where '*' denotes the convolution (on $G$ ), i.e. $(f * \mu)(s)=\int_{G} f(s / t) \mathrm{d} \mu(t)$. Moreover, $\|\Lambda\|=\|\mu\|=|\mu|(G)(|\mu|$ is the variation of $\mu)$. We easily conclude from this that $(\Phi u)(s)=\int_{0}^{\infty} \frac{u(s / t)}{t} \mathrm{~d} \mu(t)$. We shall denote the unique measure $\mu$ obtained in this way by $\mu_{\Phi}$. Note that $\left\|\mu_{\Phi}\right\|=\|\Phi\|(=\operatorname{Lip}(\Phi))$. It follows from the associativity of the convolution (on the space of signed Borel measures) that $\mu_{\Phi_{1} \circ \Phi_{2}}=\mu_{\Phi_{1}} * \mu_{\Phi_{2}}$.

Now assume that $\Phi$ is bijective and isometric and put $\mu_{1}=\mu_{\Phi}$ and $\mu_{2}=\mu_{\Phi^{-1}}$. Then we have $\left\|\mu_{1}\right\|=\left\|\mu_{2}\right\|=1$ and $\mu_{1} * \mu_{2}=\mu_{2} * \mu_{1}=\varepsilon_{1}$ where for $x \in G$, $\varepsilon_{x}$ is Dirac's measure on $G$ concentrated at $x$ (that is, $\varepsilon_{x}(A)$ is 1 if $x \in A$ and 0 otherwise). One may check that it follows from this that $\mu_{1}= \pm \varepsilon_{a}$ for some $a \in G$ and thus $\Phi(u)=a *\left( \pm \frac{1}{a} u\right)$.

(C) Let $(G, p)$ be a complete valued topological Abelian group. For each $t>0$ put $G_{t}=G$ and let $\tilde{G}$ be the direct sum of the collection $\left\{G_{t}\right\}_{t>0}$. That is, $\tilde{G}$ is a subgroup of $\prod_{t>0} G_{t}$ consisting of all functions $f:(0,+\infty) \rightarrow G$ such that the set $f^{-1}(G \backslash\{0\})$ is finite. Let $\tilde{p}: \tilde{G} \rightarrow \mathbb{R}_{+}$be defined as follows: $\tilde{p}(0)=0$; and for $f \in \tilde{G} \backslash\{0\}$ take the (unique) numbers $n \geqslant 1$ and $0<t_{1}<\ldots<t_{n}$ such that $f\left(t_{j}\right) \neq 0$ and $f(t)=0$ for $t \in(0,+\infty) \backslash\left\{t_{1}, \ldots, t_{n}\right\}$ and put $\tilde{p}(f)=$ $\sum_{j=1}^{n}\left(t_{j}-t_{j-1}\right) p\left(\sum_{k=j}^{n} f\left(t_{j}\right)\right)$ (with $\left.t_{0}=0\right)$. It is not so easy to show 'intrinsicly' that $\tilde{p}$ is a value on $\tilde{G}$. Much simpler is to check that the function $\Psi: \tilde{G} \ni f \mapsto$ $\sum_{t>0} t * \delta_{f(t)} \in L^{0}[G]$ is a group-isomorphism such that $\tilde{p}(f)=L[p](\Psi(f))$. This yields both that $\tilde{p}$ is a value on $\tilde{G}$ and that the completion $(\widehat{G}, \widehat{p})$ of $(\tilde{G}, \tilde{p})$ is isometrically isomorphic to $(L[G], L[p]$ ) (by (L3) and (L5)). This gives a more algebraic construction of $(L[G], L[p])$ for complete valued topological Abelian groups $(G, p)$. However, in this approach Lipschitz homomorphisms extend in the way which hides interesting properties (such as (L10)) of the functor because of completing the spaces ' $\tilde{G}$ '.

We infer from Example 3.6-(B) that every (bijective) linear (in the sense of the PV structure) isometry of $L[\mathbb{R}]$ is of the form $u \mapsto \frac{1}{a} * L[\varphi](u)$ where $a>0$ and $\varphi: \mathbb{R} \rightarrow \mathbb{R}$ is of the form $\varphi(t)=\varepsilon a t$ with $\varepsilon \in\{-1,1\}$. This suggests the following general assertion:

Is every linear isometry of $(L[G], L[p])$ onto $(L[H], L[q])$ of the form $u \mapsto$ $\frac{1}{a} * L[\varphi](u)$ where $a>0$ and $\varphi: G \rightarrow H$ is a group-isomorphism such that $q(\varphi(x))=\operatorname{ap}(x)$ for each $x \in G$ ?

Affirmative answer for this yields that the correspondence $(G, p) \leftrightarrow(L[G], L[p])$ is one-to-one when two valued topological groups $(G, p)$ and $(H, q)$ are identified iff $(H, q)$ is isometrically isomorphic to $(G, a p)$ for some positive number $a$. 


\section{Urysohn Universal Space as a NTPV Boolean Group}

We begin with

Definition 4.1 An Urysohn space is a separable complete metric space $\mathbb{U}$ such that every separable metric space is isometrically embeddable in $\mathbb{U}$ and each isometry between finite subsets of $\mathbb{U}$ is extendable to an isometry of $\mathbb{U}$ onto itself.

A theorem of Urysohn [17] says that there is a unique (up to isometry) Urysohn space. He has also proved that the completion of the so-called rational Urysohn space $\mathbb{Q} \mathbb{U}$ is an Urysohn space. To put the definition of $\mathbb{Q} \mathbb{U}$ we need two very useful notions. Recall that a Katětov map on a metric space $(X, d)$ is any function $f: X \rightarrow \mathbb{R}_{+}$such that $|f(x)-f(y)| \leqslant d(x, y) \leqslant f(x)+f(y)$ for all $x, y \in X$. A Katětov map $f: A \rightarrow \mathbb{R}_{+}$where $A$ is a subset of $X$ is said to be trivial (in $X$ ) iff $f(a)=d(x, a)$ for each $a \in A$ and some $x \in X$. Now we can recall the definition of the rational Urysohn space. This space is uniquely determined (up to isometry) by the following three conditions: it is countable, its metric is rational-valued and every rational-valued Katětov map defined on a finite nonempty subset of it is trivial. We may simply generalize this notion to any countable subfield of $\mathbb{R}$. Namely, if $\mathbb{F}$ is such a field, by an $\mathbb{F}$-Urysohn space, denoted by $\mathbb{F} U$, we mean any metric space which is countable, has $\mathbb{F}$-valued metric and the property that every $\mathbb{F}$-valued Katětov map defined on its finite nonempty subset is trivial. Adapting the original proof of Urysohn, one easily shows that for every countable subfield $\mathbb{F}$ of $\mathbb{R}$ there exists a unique (up to isometry) $\mathbb{F}$-Urysohn space and its completion is an Urysohn space. This fact shall be applied in the sequel. The reader interested in 'Urysohn-like' spaces is referred to a survey article [10] where one can find more references dealing with this topic.

All groups in this section are Abelian We call a group $(G,+)$ Boolean iff it is of exponent 2, that is, if $x+x=0$ for each $x \in G$. In [12] we have shown that there is a unique (up to isometric isomorphism) valued Boolean group, called the Boolean Urysohn group, which is Urysohn as a metric space. One may ask whether there is a NTPV Boolean Urysohn group. In this section we shall do a little bit more than just giving an affirmative answer for the latter problem (see Theorem 4.3 below).

For need of this section, we extend the notion of pseudovector groups (over $\mathbb{R}$ ) to PV groups over subfields of $\mathbb{R}$. Namely, for a subfield $\mathbb{F}$ of $\mathbb{R}$, denote by $\mathbb{F}_{+}$the set $\mathbb{F} \cap \mathbb{R}_{+}$and call a triple $(G,+, \cdot)$ an $\mathbb{F}$-PV group if $(G,+)$ is a group and '. ' is an action $\mathbb{F}_{+} \times G \rightarrow G$ satisfying axioms (PVG2)-(PVG4) for each $t, s \in \mathbb{F}_{+}$and $x, y \in G$. In a similar way as it was done in Section 1 we define a topological $\mathbb{F}-P V$ group (briefly, an $\mathbb{F}$-TPVG), a normed $\mathbb{F}$-PV group (an $\mathbb{F}$-NPVG) and a normed topological $\mathbb{F}$ - $P V$ group (an $\mathbb{F}$-NTPVG). (Note that the norm of an $\mathbb{F}-\mathrm{NPVG}$ does not need to take values in $\mathbb{F}_{+}$.) Everywhere below $\mathbb{F}$ denotes an arbitrary subfield of $\mathbb{R}$.

Definition 4.2 A pseudovector $v$ of an $\mathbb{F}$-NPV Abelian group $(G,\|\cdot\|)$ is said to be Lipschitz if there is a positive constant $C$ such that

$$
\|t \cdot v-s \cdot v\| \leqslant C|t-s|
$$


for each $t, s \in \mathbb{F}_{+}$. The set of all Lipschitz pseudovectors in $G$ is denoted by $\mathcal{L}(G)$. The NPV group $G$ is Lipschitz [almost Lipschitz] if $\mathcal{L}(G)=G$ [if $\mathcal{L}(G)$ is dense in $G$ ]. Additionally, $G$ is uniformly Lipschitz provided there is a universal positive constant $C$ such that Eq. 4.1 is fulfilled for every $v \in G$ with $\|v\| \leqslant 1$ and each $t, s \in \mathbb{F}_{+}$.

The reader will easily check that $\mathcal{L}(G)$ is always an $\mathbb{F}$-PV subgroup of an $\mathbb{F}$-NPV group $G$. The NTPV group $(L[G], L[p])$ of a valued Abelian group $(G, p)$ is almost Lipschitz (indeed, $L^{0}[G] \subset \mathcal{L}(L[G])$ ). One may also show, using Baire's theorem, that a Lipschitz complete NPVG is uniformly Lipschitz. At this moment, observe that-thanks to Proposition 1.4-every almost Lipschitz NPVG is a TPVG. The aim of this section is to prove the following

Theorem 4.3 Every separable almost Lipschitz NPV Boolean group can be embedded (by means of an isometric linear homomorphism) in some almost Lipschitz NPV Boolean group which is Urysohn as a metric space.

The above result implies, of course, that there is an almost Lipschitz NPV Boolean Urysohn group. This shows that the Urysohn universal space can be endowed with a structure similar to the structures of Banach spaces (it is however nonisometric to any Banach space).

The proof of Theorem 4.3 involves the next two lemmas. The proof of the first of them is left as an exercise.

Lemma 4.4 The completion $\bar{G}$ of a Lipschitz. $\mathbb{F}-N P V$ Abelian group $\bar{G}$ admits a unique structure (i.e. the addition, the multiplication by nonnegative reals and the norm) of an almost Lipschitz. NPVG which extends the $\mathbb{F}-N P V$ structure of $G$. Moreover, $G \subset \mathcal{L}(\bar{G})$.

Lemma 4.5 Suppose $\mathbb{F}$ is countable and $\left(G,\|\cdot\|_{G}\right)$ is a countable Lipschitz $\mathbb{F}-N P V$ Boolean group. There is a countable subfield $\mathbb{K}$ of $\mathbb{R}$ with $\mathbb{K} \supset \mathbb{F}$ and a Lipschitz $\mathbb{K}-N P V$ Boolean group $\left(H,\|\cdot\|_{H}\right)$ such that $G$ admits an isometric $\mathbb{F}$-linear homomorphic embedding into $H$ and $H$ is $\mathbb{K}$-Urysohn as a metric space.

For showing Lemma 4.5, we need some auxiliary sublemmas.

Sublemma 4.6 Let $\left(G,\|\cdot\|_{G}\right)$ be a Lipschitz $\mathbb{F}-N P V G$. For every Katětov map $f: A \rightarrow \mathbb{R}_{+}$where $A$ is a finite nonempty subset of $G$ there is a nonnegative constant $M(f)$ such that for every $t, s \in \mathbb{F}_{+}$and $x, y \in A$ :

$$
\|t \cdot x-s \cdot y\|_{G}-t f(x)-s f(y) \leqslant M(f)|t-s| .
$$

Proof Replacing $G$ by its completion and using Lemma 4.4, we may assume that $\mathbb{F}=$ $\mathbb{R}$ and $A$ is a subset of $\mathcal{L}(G)$. Fix $x, y \in A$ and put $\varphi_{x, y}:[0,1) \ni t \mapsto \frac{\|x-t \cdot y\|-f(x)-t f(y)}{1-t} \in$ $\mathbb{R}$. We shall show that $\varphi_{x, y}$ is upper bounded. Since it is continuous, it suffices to check that

$$
\limsup _{t \rightarrow 1^{-}} \varphi_{x, y}(t)<+\infty .
$$


Remembering that $\|x-y\| \leqslant f(x)+f(y)$, we obtain

$$
\begin{aligned}
\varphi_{x, y}(t) & =f(y)+\frac{\|x-y\|-f(x)-f(y)}{1-t}+\frac{\|x-t \cdot y\|-\|x-y\|}{1-t} \\
& \leqslant f(y)+\frac{\|y-t \cdot y\|}{1-t} \leqslant f(y)+C
\end{aligned}
$$

where $C$ is as in Eq. 4.1 with $v=y$. This gives Eq. 4.3. Now it is enough to put $M(f)=$ $\max \left\{\sup \varphi_{x, y}([0,1)): x, y \in A\right\}$ to end the proof.

Sublemma 4.7 Suppose $\mathbb{F}$ is countable, $\left(G,\|\cdot\|_{G}\right)$ is a countable Lipschitz $\mathbb{F}-N P V$ Boolean group and $f: A \rightarrow \mathbb{R}_{+}$is a Katětov map defined on a finite nonempty subset $A$ of $G$. The $\mathbb{F}-N P V G\left(G,\|\cdot\|_{G}\right)$ can be enlarged to a countable Lipschitz $\mathbb{F}-N P V$ Boolean group $\left(H,\|\cdot\|_{H}\right)$ such that $f$ is trivial in $H$.

Proof Let us agree that $\sum_{j=1}^{0}=0$. Take a constant $M(f)$ appearing in Sublemma 4.6 and put $M=\max (M(f)$, $\max f(A))$. Let $\left(\mathfrak{B},\|\cdot\|_{0}\right)$ be as in Example 1.6. Evidently, $\mathfrak{B}$ is an $\mathbb{F}$-PV (after restricting the multiplication). Let $e=\{1\}, E=\operatorname{lin}_{\mathbb{F}}\{1\} \subset \mathfrak{B}$ (that is, $E$ is the smallest $\mathbb{F}$-PV subgroup of $\mathfrak{B}$ containing $\{1\}$ ) and let $\|\cdot\|_{E}$ be the restriction of $\|\cdot\|_{0}$ to $E$. Put $\widehat{H}=G \oplus E$ and define a function $\|\cdot\|: \widehat{H} \rightarrow \mathbb{R}_{+}$by

$$
\begin{array}{r}
\|x \oplus y\|=\inf \left\{\left\|x+\sum_{j=1}^{n} t_{j} \cdot a_{j}\right\|_{G}+\sum_{j=1}^{n} t_{j} f\left(a_{j}\right)+M\left\|y+\sum_{j=1}^{n} t_{j} \cdot e\right\|_{E}:\right. \\
\left.n \geqslant 0, t_{1}, \ldots, t_{n} \in \mathbb{F}_{+}, t_{1}<\ldots<t_{n}, a_{1}, \ldots, a_{n} \in A\right\}
\end{array}
$$

for $x \in G$ and $y \in E$. The group $\widehat{H}$ is clearly Boolean and $\mathbb{F}$-PV. We shall prove that:

(AUX1) $\|\cdot\|$ is a seminorm on $\widehat{H}$,

(AUX2) $\|x \oplus 0\|=\|x\|_{G}$ for any $x \in G$,

(AUX3) $\|a \oplus e\|=f(a)$ for each $a \in A$.

To establish (AUX1), it is enough to check the triangle inequality. So, let $x^{\prime}$, $x^{\prime \prime} \in G, y^{\prime}, y^{\prime \prime} \in E$, and $n, m \geqslant 0, t_{1}, \ldots, t_{n}, s_{1}, \ldots, s_{m} \in \mathbb{F}_{+}$be such that $t_{1}<\ldots<t_{n}$ and $s_{1}<\ldots<s_{m}$ and let $a_{1}, \ldots, a_{n}$ and $b_{1}, \ldots, b_{m}$ belong to $A$. All elements of the set $\left\{t_{1}, \ldots, t_{n}, s_{1}, \ldots, s_{m}\right\}$ arrange in a sequence $p_{1}<\ldots<p_{r}$ and let $J$ consists of all those $j \in\{1, \ldots, r\}$ that $p_{j} \in\left\{t_{1}, \ldots, t_{n}\right\} \cap\left\{s_{1}, \ldots, s_{m}\right\}$. For any $j \in J$ there are unique $\alpha(j) \in\{1, \ldots, n\}$ and $\beta(j) \in\{1, \ldots, m\}$ with $p_{j}=t_{\alpha(j)}=s_{\beta(j)}$. After erasing all $p_{j}$ 's with $j \in J$ from the system $p_{1}, \ldots, p_{r}$ we obtain numbers $q_{1}<\ldots<q_{d}$ and corresponding to them points $c_{1}, \ldots, c_{d}$ of $A$ by the rule: $c_{k}=a_{k}$ iff $q_{k}=t_{k}$ and $c_{k}=b_{k}$ iff $q_{k}=s_{k}$. Now we have $\left\|\left(x^{\prime}+x^{\prime \prime}\right) \oplus\left(y^{\prime}+y^{\prime \prime}\right)\right\| \leqslant \| x^{\prime}+x^{\prime \prime}+$ $\sum_{k=1}^{d} q_{k} \cdot c_{k}\left\|_{G}+\sum_{k=1}^{d} q_{k} f\left(c_{k}\right)+M\right\| y^{\prime}+y^{\prime \prime}+\sum_{k=1}^{d} q_{k} \cdot e \|_{E}$ and the right hand side expression in this inequality is equal to $\|\left(x^{\prime}+\sum_{j=1}^{n} t_{j} \cdot a_{j}\right)+\left(x^{\prime \prime}+\sum_{j=1}^{m} s_{j} \cdot b_{j}\right)+$ $\sum_{j \in J} p_{j} \cdot\left(a_{\alpha(j)}+b_{\beta(j)}\right)\left\|_{G}+\sum_{k=1}^{d} q_{k} f\left(c_{k}\right)+M\right\|\left(y^{\prime}+\sum_{j=1}^{n} t_{j} \cdot e\right)+\left(y^{\prime \prime}+\sum_{j=1}^{m} s_{j} \cdot e\right) \|_{E}$ which-by the triangle inequality-is no greater than $\left\|x^{\prime}+\sum_{j=1}^{n} t_{j} \cdot a_{j}\right\|_{G}+\| x^{\prime \prime}+$ $\sum_{j=1}^{m} s_{j} \cdot b_{j}\left\|_{G}+\sum_{j \in J} p_{j}\right\| a_{\alpha(j)}+b_{\beta(j)}\left\|_{G}+\sum_{k=1}^{d} q_{k} f\left(c_{k}\right)+M\right\| y^{\prime}+\sum_{j=1}^{n} t_{j} \cdot e\left\|_{E}+M\right\| y^{\prime \prime}+$ $\sum_{j=1}^{m} s_{j} \cdot e \|_{E}$. These notices combined with the fact that $\left\|a_{\alpha(j)}+b_{\beta(j)}\right\|_{G} \leqslant f\left(a_{\alpha(j)}\right)+$ 
$f\left(b_{\beta(j)}\right)$ for each $j \in J$ yield $\left\|\left(x^{\prime}+x^{\prime \prime}\right) \oplus\left(y^{\prime}+y^{\prime \prime}\right)\right\| \leqslant\left\|x^{\prime}+\sum_{j=1}^{n} t_{j} \cdot a_{j}\right\|_{G}+\sum_{j=1}^{n} t_{j} f\left(a_{j}\right)+$ $M\left\|y^{\prime}+\sum_{j=1}^{n} t_{j} \cdot e\right\|_{E}+\left\|x^{\prime \prime}+\sum_{j=1}^{m} s_{j} \cdot b_{j}\right\|_{G}+\sum_{j=1}^{m} s_{j} f\left(b_{j}\right)+M\left\|y^{\prime \prime}+\sum_{j=1}^{m} s_{j} \cdot e\right\|_{E}$, which is equivalent to the triangle inequality. To show (AUX2) and (AUX3), we only need to prove the inequalities ' $\geqslant$ '.

To establish (AUX2), we have to show that

$$
\|x\|_{G} \leqslant\left\|x+\sum_{j=1}^{n} t_{j} \cdot a_{j}\right\|_{G}+\sum_{j=1}^{n} t_{j} f\left(a_{j}\right)+M\left\|\sum_{j=1}^{n} t_{j} \cdot e\right\|_{E}
$$

for each $n \geqslant 1, t_{1}, \ldots, t_{n} \in \mathbb{F}_{+}$with $t_{1}<\ldots<t_{n}$ and every $a_{1}, \ldots, a_{n} \in A$. Enlarging, if needed, the system $t_{1}, \ldots, t_{n}$ to $0, t_{1}, \ldots, t_{n}$, we may assume that $n$ is even, say $n=2 m$. But then $\left\|\sum_{j=1}^{n} t_{j} \cdot e\right\|_{E}=\sum_{j=1}^{m}\left(t_{2 j}-t_{2 j-1}\right)$. Therefore Eq. 4.4 is implied by

$$
\begin{aligned}
& \sum_{j=1}^{m}\left\|t_{2 j-1} \cdot a_{2 j-1}+t_{2 j} \cdot a_{2 j}\right\|_{G} \leqslant \\
& \quad \leqslant \sum_{j=1}^{m}\left(t_{2 j-1} f\left(a_{2 j-1}\right)+t_{2 j} f\left(a_{2 j}\right)\right)+M \sum_{j=1}^{m}\left|t_{2 j-1}-t_{2 j}\right|
\end{aligned}
$$

which is fulfilled because $M \geqslant M(f)$.

We pass to (AUX3). Fix $a \in A$. As before, take any $n \geqslant 1, a_{1}, \ldots, a_{n} \in A$ and $t_{1}, \ldots, t_{n} \in \mathbb{F}_{+}$with $t_{1}<\ldots<t_{n}$. We need to show that

$$
f(a) \leqslant\left\|a+\sum_{j=1}^{n} t_{j} \cdot a_{j}\right\|_{G}+\sum_{j=1}^{n} t_{j} f\left(a_{j}\right)+M\left\|e+\sum_{j=1}^{n} t_{j} \cdot e\right\|_{E} .
$$

First assume that there is $p \in\{1, \ldots, n\}$ with $t_{p}=1$. In that case Eq. 4.5 follows from the inequality $\left\|a+a_{p}\right\|_{G} \leqslant\left\|\left(a+a_{p}\right)+\sum_{j \neq p} t_{j} \cdot a_{j}\right\|_{G}+\sum_{j \neq p} t_{j} f\left(a_{j}\right)+M \| \sum_{j \neq p} t_{j}$. $e \|_{E}$ which is covered by (AUX2).

Now consider the case when $t_{j} \neq 1$ for each $j$. Enlarging, if needed, the system $t_{1}, \ldots, t_{n}$ to $0, t_{1}, \ldots, t_{n}$, we may assume that $n$ is odd. By the definition of the norm on $E$, there is $q \in\{1, \ldots, n\}$ such that

$$
\left\|e+\sum_{j=1}^{n} t_{j} \cdot e\right\|_{E}=\left|t_{q}-1\right|+\left\|\sum_{j \neq q} t_{j} \cdot e\right\|_{E} .
$$

Then we have $f(a) \leqslant\left\|a+a_{q}\right\|_{G}+f\left(a_{q}\right) \leqslant\left\|a+\sum_{j=1}^{n} t_{j} \cdot a_{j}\right\|_{G}+\left\|\sum_{j \neq q} t_{j} \cdot a_{j}\right\|_{G}+$ $\sum_{j=1}^{n} t_{j} f\left(a_{j}\right)+\left(1-t_{q}\right) f\left(a_{q}\right)-\sum_{j \neq q} t_{j} f\left(a_{j}\right)$. Thus, thanks to Eq. 4.6 and the fact that $M \geqslant f\left(a_{q}\right)$, to prove Eq. 4.5 it suffices to show that $\left\|\sum_{j \neq q} t_{j} \cdot a_{j}\right\|_{G}-\sum_{j \neq q} t_{j} f\left(a_{j}\right) \leqslant$ $M\left\|\sum_{j \neq q} t_{j} \cdot e\right\|_{E}$. The latter inequality is fulfilled because of the form of the norm $\|\cdot\|_{E}$ and the following two facts: $M \geqslant M(f)$ and $n$ is odd.

Having (AUX1)-(AUX3), define $H$ as the quotient $\mathbb{F}$-PV group $\widehat{H} / N$ with $N=\{h \in \widehat{H}:\|h\|=0\}$ and put $\|[h]\|_{H}=\|h\|$ for every $h \in \widehat{H}$. It is easily seen that $\left(H,\|\cdot\|_{H}\right)$ is a countable $\mathbb{F}$-NPV Boolean group. What is more, (AUX2) implies that the function $G \ni x \mapsto[x \oplus 0] \in H$ is an isometric linear homomorphism and thus we may identify $x$ with $[x \oplus 0]$ so that $G$ is a $\mathbb{F}$-PV subgroup of $H$. We infer from (AUX3) that under such an identification $f$ is trivial in $H$. It remains to check that $H$ is Lipschitz. This follows since $\mathcal{L}(H)$ is an $\mathbb{F}$-PV subgroup of $H$ and $G \cup\{[0 \oplus e]\} \subset$ $\mathcal{L}(H)$ (note that $\left.\|t \cdot[0 \oplus e]+s \cdot[0 \oplus e]\|_{H} \leqslant\|t \cdot e+s \cdot e\|_{E}=|t-s|\right)$. 
Noticing that the family of all $\mathbb{F}$-valued Katětov maps whose domains are finite nonempty subsets of $G$ is countable provided so are $\mathbb{F}$ and $G$, a simple use of Sublemma 4.7 and inductive argument give

Sublemma 4.8 Suppose $\mathbb{F}$ is countable. Every countable Lipschitz $\mathbb{F}-N P V$ Boolean group $G$ can be enlarged to a countable Lipschitz $\mathbb{F}-N P V$ Boolean group $H$ such that each $\mathbb{F}$-valued Katětov map whose domain is a finite nonempty subset of $G$ is trivial in $H$.

Proof of Lemma 4.5 Start with $\mathbb{F}_{0}=\mathbb{F}$ and $\left(H_{0},\|\cdot\|_{0}\right)=\left(G,\|\cdot\|_{G}\right)$ and assume that for some $n \geqslant 1$ we have defined a countable subfield $\mathbb{F}_{n-1}$ of $\mathbb{R}$ and a countable Lipschitz $\mathbb{F}_{n-1}$-NPV Boolean group $\left(H_{n-1},\|\cdot\|_{n-1}\right)$. By Lemma 4.4, we may assume that $H_{n-1} \subset \mathcal{L}\left(G_{n}^{\prime}\right)$ for some NPV Boolean group $\left(G_{n}^{\prime},\|\cdot\|_{n}^{\prime}\right)$ (such that the NPVstructure of $G_{n}^{\prime}$ extends the $\mathbb{F}_{n-1}$-NPV structure of $\left.H_{n-1}\right)$. Let $\mathbb{F}_{n}$ be the smallest subfield of $\mathbb{R}$ containing $\mathbb{F}_{n-1}$ and the image of $\|\cdot\|_{n-1}$ and put $G_{n}=\operatorname{lin}_{\mathbb{F}_{n}} H_{n-1} \subset G_{n}^{\prime}$. Now apply Sublemma 4.8 to obtain a countable Lipschitz $\mathbb{F}_{n}$-NPV Boolean group $\left(H_{n},\|\cdot\|_{n}\right)$ such that $H_{n}$ contains $G_{n}$ and every $\mathbb{F}_{n}$-valued Katětov map defined on a finite nonempty subset of $G_{n}$ is trivial in $H_{n}$.

The above construction ensures us that $\mathbb{K}=\bigcup_{n=0}^{\infty} \mathbb{F}_{n}$ and

$$
\left(H,\|\cdot\|_{H}\right)=\bigcup_{n=0}^{\infty}\left(H_{n},\|\cdot\|_{n}\right)
$$

are well defined and satisfy all requirements of the lemma.

Proof of Theorem 4.3 Let $\left(G,\|\cdot\|_{G}\right)$ be a separable almost Lipschitz NPV Boolean group. By Corollary 1.5, we may assume that $G$ is complete. Take a countable dense subset $A$ of $\mathcal{L}(G)$ and put $H=\operatorname{lin}_{\mathbb{Q}} A$. Thanks to Lemma 4.4, it suffices to show that $H$ may be embedded by means of an isometric $\mathbb{Q}$-linear homomorphism in an almost Lipschitz NPV Boolean Urysohn group. But this follows from Lemma 4.5, Lemma 4.4 and the fact that the completion of $\mathbb{F} U$ is Urysohn for every countable subfield $\mathbb{F}$ of $\mathbb{R}$.

We end the paper with the following problems dealing with the subjects discussed in this section.

Question 1. Are there (at least) two nonisomorphic almost Lipschitz NPV Boolean Urysohn groups?

Question 2. Is there a Lipschitz NPV Boolean Urysohn group? If yes, is it unique?

Question 3. Is $\mathbb{Q} U$ isometric to a Lipschitz $\mathbb{Q}$-NPV Boolean group?

Open Access This article is distributed under the terms of the Creative Commons Attribution Noncommercial License which permits any noncommercial use, distribution, and reproduction in any medium, provided the original author(s) and source are credited.

\section{References}

1. Berberian, S.K.: Lectures in functional analysis and operator theory. In: Graduate Texts in Mathematics, vol. 15. Springer, New York (1974) 
2. Bessaga, Cz., Pełczyński, A.: Selected Topics in Infinite-Dimensional Topology. PWN—Polish Scientific Publishers, Warszawa (1975)

3. Birkhoff, G.: A note on topological groups. Compos. Math. 3, 427-430 (1936)

4. Bruckner, A.M., Bruckner, J.B., Thomson, B.S.: Real Analysis. Prentice-Hall, New Jersey (1997)

5. Dugundji, J.: An extension of Tietze's theorem. Pac. J. Math. 1, 353-367 (1951)

6. Hoffmann, B.: A compact contractible topological group is trivial. Arch. Math. 32, 585-587 (1979)

7. Holmes, M.R.: The Urysohn space embeds in Banach spaces in just one way. Topol. Appl. 155, 1479-1482 (2008)

8. Kakutani, S.: Über die Metrisation der topologischen Gruppen. Proc. Imp. Acad. Tokyo 12, $82-$ 84 (1936)

9. Klee, Jr., V.L.: Invariant metrics in groups (solution of a problem of Banach). Proc. Am. Math. Soc. 3, 484-487 (1952)

10. Melleray, J.: Some geometric and dynamical properties of the Urysohn space. Topol. Appl. 155, 1531-1560 (2008)

11. Melleray, J., Petrov, F.V., Vershik, A.M.: Linearly rigid metric spaces and the embedding problem. Fundam. Math. 199, 177-194 (2008)

12. Niemiec, P.: Urysohn universal spaces as metric groups of exponent 2. Fundam. Math. 204, 1-6 (2009)

13. Pazy, A.: Semigroups of Linear Operators and Applications to Partial Differential Equations. Applied Mathematical Sciences, vol. 44. Springer, New York (1983)

14. Rudin, W.: Fourier Analysis on Groups. Interscience Tracts in Pure and Applied Mathematics, Number 12. Interscience Publishers, New York (1962)

15. Toruńczyk, H.: Characterizing Hilbert space topology. Fundam. Math. 111, 247-262 (1981)

16. Toruńczyk, H.: A correction of two papers concerning Hilbert manifolds. Fundam. Math. 125, 89-93 (1985)

17. Urysohn, P.S.: Sur un espace métrique universel. Bull. Sci. Math. 51, 43-64, 74-96 (1927) 